Pensamiento Crítico Vol. 18 № 2, pp. 105-113

\title{
Demanda de dinero intertemporal con generaciones yuxtapuestas
}

Richard Roca ${ }^{1}$

\section{RESUMEN}

El presente trabajo expone la teoría de demanda de dinero intertemporal de generaciones yuxtapuestas de Samuelson en la que en cada periodo coexisten diferentes generaciones que se van sucediendo en el tiempo y cada individuo tiene una vida limitada. El dinero es el único activo duradero que los agentes pueden mantener para poder comprar cuando estén en la etapa de retiro.

Palabras claves: Demanda de dinero, Generaciones yuxtapuestas, dinero intertemporal, Samuelson, modelos económicos.

\begin{abstract}
This paper presents the Samuelson's Model of Overlapping Generations demand for money in wich each period coexist different generations with limited life. Money is the only lasting asset that agents can maintain to purchase when they are in the retirement stage.
\end{abstract}

Keywords: Money demand, Overlapping Generations, intertemporal money, Samuelson, economic models.

1 Profesor Principal de Economía de la Universidad Nacional Mayor de San Marcos.

Correo electrónico: rhroca@yahoo.com 


\section{Pensamiento Crítico Vol. 18. № 2}

\section{INTRODUCCIÓN}

Esta teoría, desarrollada por Samuelson (1958), considera la demanda de dinero como un activo duradero, como una reserva de valor que le permite a las personas guardar poder de compra durante mucho tiempo en un marco intertemporal en el que coexisten diferentes generaciones que tiene vidas limitadas y se van sucediendo en el tiempo.

\section{EL MODELO}

En la versión simple que se presenta a continuación se supone que cada persona vive solo dos periodos: 1 juventud, 2 vejez o retiro. En cada periodo coexisten dos generaciones: jóvenes y viejos. Cada joven produce un bien homogéneo no almacenable: $y_{1, t}$ además consume bienes: $c_{1, t}$. Los viejos están jubilados, solo consumen. $C_{2, t+1}$

La producción total del periodo t:

$$
Y_{t}=N_{1 t} y_{1 t}
$$

La restricción presupuestaria del periodo t:

$$
N_{1 t} c_{1 t}+N_{2 t} c_{2 t} \leq N_{1 t} y
$$

Cada joven tiene $1+n$ hijos: tasa de crecimiento poblacional: $n$.

\section{Equilibrio en una Economía de Trueque}

Si se reparte toda la producción de los jóvenes entre jóvenes y viejos:

$$
c_{2 t}=\frac{N_{1 t}}{N_{2 t}} y-\frac{N_{1 t}}{N_{2 t}} c_{1 t}
$$

Dado que cada joven tiene $1+n$ hijos, $N_{1 t}=(1+n) N_{2 t}$ :

$$
c_{2 t}=(1+n) y-(1+n) c_{1 t}
$$




\section{Richard Roca}

Si toda la producción es consumida por los jóvenes lo máximo que podría consumir cada joven seria: $c_{1 t}=y$. Si toda la producción se entregara a los ancianos lo máximo que podría consumir cada anciano seria: $c_{2 t}=(1+n) y$.

FIGURA 1. Restricción Presupuestaria de la Sociedad en cada periodo t.

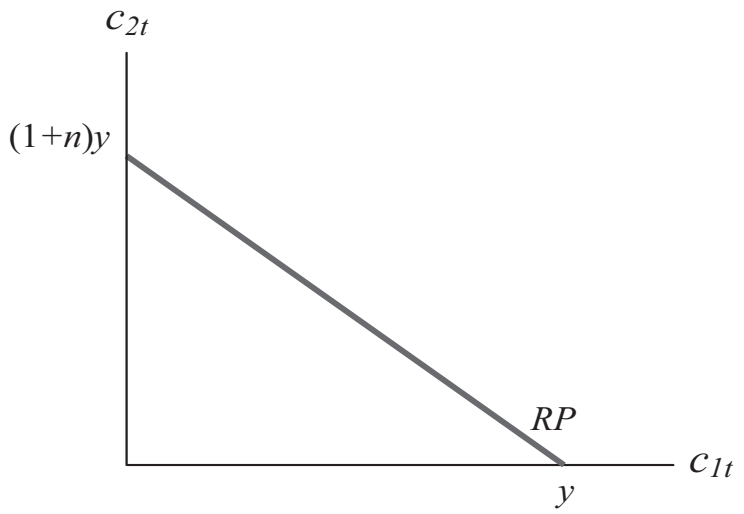

Con una producción y población creciendo establemente cada individuo de cada periodo tendría la misma restricción presupuestaria.

FIGURA 2. Restricción presupuestaria intertemporal individual

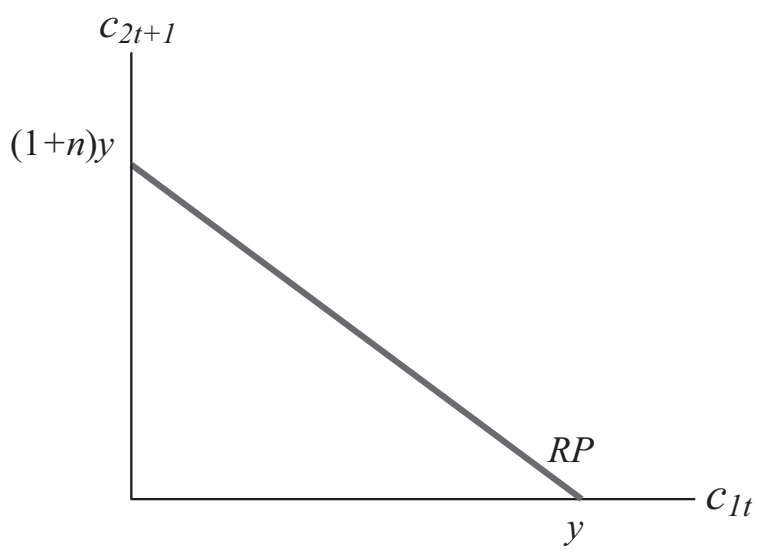




\section{Pensamiento Crítico Vol. 18. No 2}

Cada persona tratará de maximizar el valor presente del bienestar de toda su vida:

$$
U=U\left(c_{1 t}, c_{2 t+1}\right)
$$

Se supone utilidad marginal decreciente: $U_{1}>0>U_{11}, U_{2}>0>U_{22}$ y que las utilidades marginales son complementarias $U_{12}>0<U_{21}$ lo que nos daría curvas de indiferencia convexas al origen como se muestran en la figura 3.

FIGURA 3. Las preferencias intertemporales de cada individuo

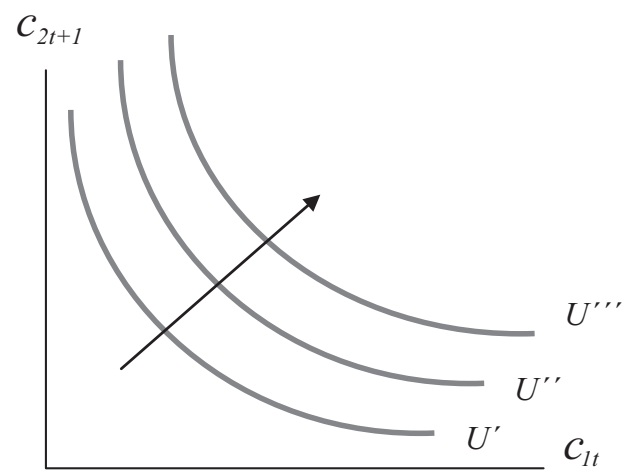

Un individuo trataría de poder consumir a lo largo de toda su vida y tener una canasta intertemporal como del punto $C$ de la figura 4.

FIGURA 4. Las preferencias intertemporales y la RP de cada individuo.

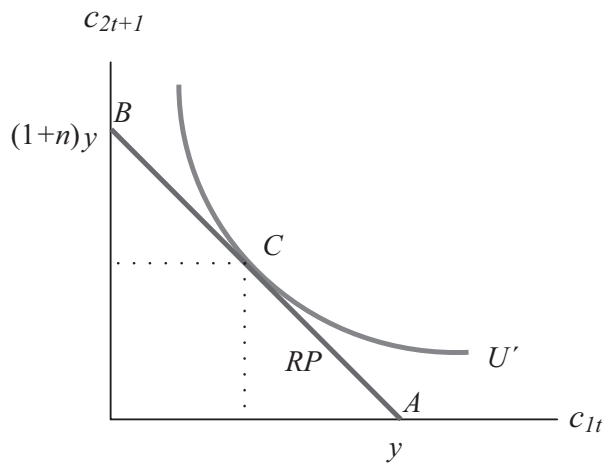




\section{Richard Roca}

Lo que involucra un intercambio voluntario entre jóvenes y viejos. Pero los viejos no tienen nada que ofrecer a cambio a los jóvenes. No serviría el crédito. En el siguiente periodo los viejos están muertos.

El equilibrio de trueque competitivo es el punto $\mathrm{A}$, de la figura 4, cada joven consume todo lo que produce en su juventud y nada en la vejez. El equilibrio de trueque competitivo no es óptimo de Pareto.

\section{Economía monetaria}

Supongamos que el gobierno dona a los viejos $H$ unidades monetarias, perfectamente divisibles y almacenables, que los usan para comprar bienes a los jóvenes.

Si cada persona acepta el dinero creyendo que puede cambiarlo por bienes más adelante, cada persona en su juventud puede vender parte de su producción a cambio de dinero:

En el periodo 1:

$$
P_{t}\left(y-c_{1 t}\right)=M_{t}^{d}
$$

Lo guarda para la vejez y compra bienes con dicho dinero a los jóvenes del siguiente periodo.

En el periodo 2:

$$
P_{t+1} c_{2 t+1}<M_{t}^{d}
$$

si no deja dinero sin gastar al dinero al final de vida:

$$
c_{2 t+1}=\frac{P_{t}}{P_{t+1}} \frac{M_{t}^{d}}{P_{t}}=\frac{1}{1+\pi_{t+1}} m_{t}^{d}
$$




\section{Pensamiento Crítico Vol. 18. $N^{\circ} 2$}

Combinando ambas restricciones obtenemos la restricción presupuestaria:

$$
c_{2 t+1}=\frac{P_{t}}{P_{t+1}}\left(y-c_{1 t}\right)
$$

Cada persona tratará de maximizar su utilidad intertemporal lo que se daría, gráficamente, en un punto como $C$ de la figura 5 . El producto no consumido es vendido a cambio de dinero. La diferencia entre el producto el consumo óptimo del primer periodo es la demanda óptima de dinero $m_{t}$.

FIGURA 5. Equilibrio intertemporal y demanda de dinero

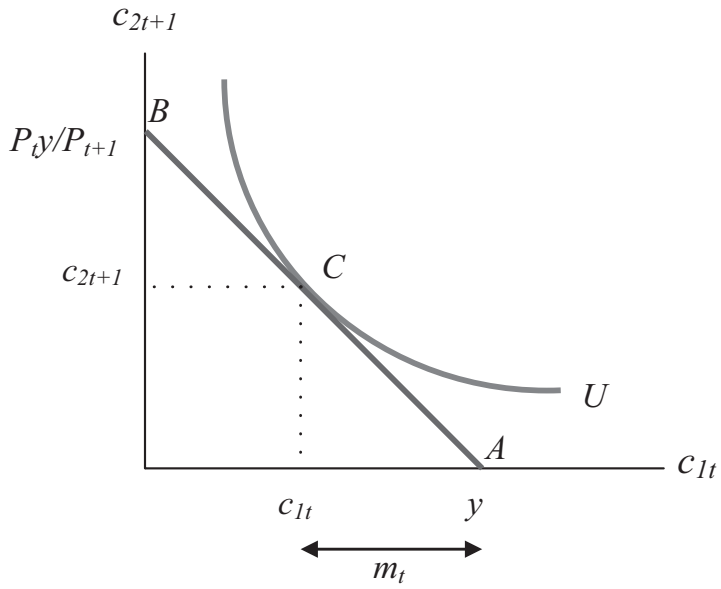

Condición de maximización:

$$
\frac{U_{1}\left(c_{1}, c_{2}\right)}{U_{2}\left(c_{1}, c_{2}\right)}=\frac{P_{t}}{P_{t+1}}
$$

o, lo que es lo mismo:

$$
\frac{U_{1}\left(c_{1}, c_{2}\right)}{U_{2}\left(c_{1}, c_{2}\right)}=\frac{1}{1+\pi_{t+1}}
$$




\section{Richard Roca}

Considerando $\mathrm{t}=1$ :

$$
\begin{gathered}
\frac{U_{1}\left(c_{1}, c_{2}\right)}{U_{2}\left(c_{1}, c_{2}\right)}=\frac{1}{1+\pi_{2}} \\
U_{1}\left(c_{1}, c_{2}\right)\left(1+\pi_{2}\right)=U_{2}\left(c_{1}, c_{2}\right)
\end{gathered}
$$

diferenciando y reordenando la anterior:

$\left[U_{11}\left(1+\pi_{2}\right)-U_{21}\right] d c_{1}+\left[U_{12}\left(1+\pi_{2}\right)-U_{22}\right] d c_{2}=-U_{1} d \pi_{2}$

de las restricciones presupuestarias:

$c_{1}=y-m_{1}, c_{2}=\frac{1}{1+\pi_{2}} m_{1}^{d}$

en (4):

$\left[U_{11}\left(1+\pi_{2}\right)-U_{21}\right] d\left(y_{1}-m_{1}\right)+\left[U_{12}\left(1+\pi_{2}\right)-U_{22}\right] d\left(\frac{1}{1+\pi_{2}} m_{1}\right)=-U_{1} d \pi_{2}$ de donde:

$$
d m_{1}=\left[\frac{\frac{U_{12}\left(1+\pi_{2}\right)-U_{22}}{\left(1+\pi_{2}\right)^{2}} m_{1}-U_{1}}{-U_{11}\left(1+\pi_{2}\right)+U_{21}+U_{12}-\frac{U_{22}}{1+\pi_{2}}}\right] d \pi_{2}+\left[\frac{-U_{11}\left(1+\pi_{2}\right)+U_{21}}{-U_{11}\left(1+\pi_{2}\right)+U_{21}+U_{12}-\frac{U_{22}}{1+\pi_{2}}}\right] d y_{1}
$$

Analizando los signos del coeficiente del diferencial de la inflación esperada se obtiene que tiene signo indeterminado pues mientras que el numerador tiene signo indeterminado el denominador tiene signo positivo. Ello refleja el hecho de que el efecto renta esta en contra del efecto sustitución lo que normalmente se presenta en el caso del ahorro. Aquí el dinero es un medio de ahorro. El signo del coeficiente del diferencial del ingreso real es definidamente positivo: 


\section{Pensamiento Crítico Vol. 18. № 2}

$$
d m_{1}^{d}=\left[\frac{(+,-)}{(+)}\right] d \pi_{2}+\left[\frac{(+)}{(+)}\right] d y_{1}
$$

de donde:

$$
m_{i}^{d}=\begin{gathered}
m\left(\pi_{2}, y_{1}\right) \\
+,-
\end{gathered}
$$

En la figura 6 se muestra el efecto de un aumento del nivel de precios esperado para el siguiente periodo lo que hace rotar la recta presupuestaria intertemporal, en sentido anti horario, a $R P_{2}$. El punto 1' muestra el efecto sustitución del aumento de precio futuro esperado, de acuerdo al cual la demanda real de dinero se reduce. Además, se genera un efecto renta, el desplazamiento paralelo de la $R P$ hacia el origen. Suponiendo que tanto el consumo presente como el futuro son bienes normales se tendría que reducir ambos por lo que el punto final puede ser tanto un punto como 2 , a la derecha del punto 1, o un punto como 2' a la izquierda del punto 1 . El punto 2 implica una menor demanda real de dinero respecto al punto 1 (efecto sustitución mas fuerte que el efecto renta). Pero el punto 2' (si el efecto renta es mas fuerte que el efecto sustitución) implica que la demanda real de dinero hubiera aumentado.

FIGURA 6. Aumento del nivel de precio esperado y la demanda de dinero

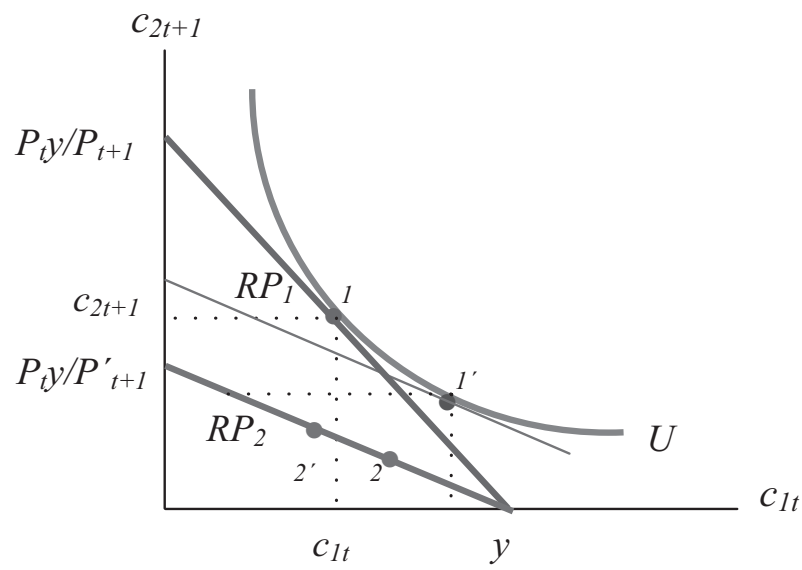




\section{Richard Roca}

Una reducción del nivel de producción de los jóvenes reduce tanto el consumo presente como la demanda real de dinero como se observa en la figura 8 .

FIGURA 8. Caída del nivel de producción y la demanda de dinero.

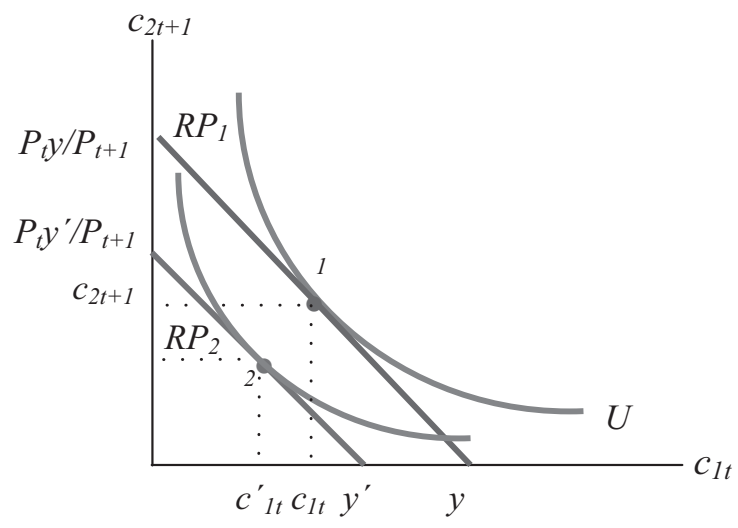

\section{CONCLUSIONES}

El modelo presentado explica la demanda por dinero como una reserva de valor que permite a los individuos guardar poder de compra en el tiempo, como un medio de ahorro, para cuando lleguen a la etapa de retiro en la que no puedan trabajar. El dinero es el único medio de ahorro lo que pondría en aprietos a esta teoría si aparece otros activos duraderos como los bonos que tendrían mayor rentabilidad o si se usa a algunos bienes como la tierra o los metales.

\section{REFERENCIAS}

Blanchard, O. y S. Fischer (1989). Lectures on Macroeconomics. Mit Press.

Champ B. y S. Freeman (1994). Modelling Monetary Economies. Wiley \& Sons. INC.

Samuelson, P.A. (1958). An exact model of loan consumption on interest whit or without the social contrivance of money. Journal of Political Economy. Diciembre. 